\title{
Oblique illumination in microscopy: a quantitative evaluation
}

\author{
Carlos Sanchez ${ }^{\mathrm{a}}$, Gabriel Cristóbal $^{\mathrm{a}}$, Gloria Bueno ${ }^{\mathrm{b}}$, Saul Blanco $^{\mathrm{c}}$, Maria Borrego-Ramos $^{\mathrm{c}}$, Adriana Olenici $^{\mathrm{c}, \mathrm{d}}$, Anibal Pedraza $^{\mathrm{b}}$, \\ Jesus Ruiz-Santaquiteriab
}

${ }^{a}$ Instituto de Óptica (CSIC), Serrano 121, 28006 Madrid, Spain

${ }^{b}$ Univ. Castilla La Mancha, Av. Camilo Jose Cela s/n, 13071 Ciudad Real, Spain

${ }^{c}$ Institute of the Environment, Univ. of Leon, La Serna 58, 24007 Leon, Spain

${ }^{d}$ Fac. of Environmental Sci. and Eng., Babes-Bolyai University, Cluj-Napoca, Romania

\begin{abstract}
Many biological objects are barely distinguished with the brightfield microscope because they appear transparent, translucent and colourless. One simple way to make such specimens visible without compromising contrast and resolution is by controlling the amount and the directionality of the illumination light. Oblique illumination is an old technique described by many scientists and microscopists that however has been largely neglected in favour of other alternative methods. Oblique lighting is created by illuminating the sample by only a portion of the light coming from the condenser. If properly used it can improve the resolution and contrast of transparent specimens such as diatoms. In this paper a quantitative evaluation of OL in brigthfield microscopy is presented. Several feature descriptors were selected for characterising contrast and sharpness showing that in general OL provides better performance for distinguishing minute details compared to other lighting modalities. Oblique lighting is capable to produce directionally shadowed differential contrast images allowing to observe phase details in a similar way to differential contrast images (DIC) but at lower cost. The main advantage of OL is that the resolution of the light microscope can be increased by effectively doubling the angular aperture. OL appears as a cost-effective technique both for the amateur and professional scientist that can be used as a replacement of DIC or phase contrast when resources are scarce.
\end{abstract}

Keywords: Oblique illumination; contrast; feature descriptors; resolution.

\section{Introduction}

In photography, oblique light (OL) is a technique that allows to show detail by creating shadows on the surface of the object. This is one of the reasons most of the photographers prefer to capture images at sunset or dawn. Side photography allows to emphasise texture, defining depth and bringing out pattern and detail. An old debate in microscopy was to elucidate why different illumination techniques will produce different results for different specimens. In general, it can be shown that oblique illumination improves the contrast of transparent specimens by introducing a pseudo-relief effect. Oblique illumination is an old technique that has been described by many scientists and microscopists [1-7]. Oblique lighting can be interpreted in the context of the Abbe's theory of diffraction that establishes that the finest details of the specimen are obtained from diffracted beams that are apart of the axial light [1]. According with the Abbe's theory of diffraction, at least two orders of diffraction are needed to recover the details of the object. With oblique light the objective lens capture positive diffraction orders from one side of the specimen and negative from the other side [8]. In practice, oblique illumination is obtained by blocking part of the condenser's light through the use of particular patch stops with different shapes. The main advantage of OL is that the resolution of the light microscope can be increased by effectively doubling the angular aperture. One of the inconveniences of $\mathrm{OL}$ is that it appears to improve resolution in a directional way depending on the direction of the oblique mask. A simple variation of OL is produced by inserting an opaque stop with one or several small apertures or notches on the border of the stop. This procedure generates few narrow pencils of illumination in a very oblique way [2]. Both kind of unsymmetrical OL introduce similar strong pseudo-relief effect improving the edges of the objects. Another way to improve resolution and contrast is to use an inverted oblique "hollow-cone illumination" or circular oblique lighting (COL) [9], and because COL is symmetrical it does not produce the strong pseudo-relief found with OL. In fact, as R. Wayne mentioned in his recent book "there is no reason why all microscopes should not be equipped with annular illumination as a standard feature to maximise the resolving power" [8]. It is important not to confuse COL with dark field (DF) illumination which is again a very old technique. In the latter case (not discussed in this paper), the specimen is illuminated with a hollow cone of light which is too wide to enter the objective lens. Once the object is placed on the stage only the first and higher-order diffracted light will be able to enter the objective lens [8]. DF can be interpreted as a process to remove the low-frequency components of the Fourier spectrum and therefore will become more effective for improving the edges or boundaries of the objects and less useful for the inner texture (except for patterns with high transparent detail).

The main purpose of this paper is to provide a quantitative account of OL as a simple and efficient variation in brightfield 
microscopy. Improvements in contrast and enhancing details have been evaluated through the use of texture descriptors and the quality of the images has been assessed by considering an ideal observer model. Different oblique filter sets have been designed and printed out using a 3D printer. Such designs are provided and they can be easily adapted to different microscope configurations [10]. This paper is structured as follows. The details about the materials and the equipment used are described in section 2. Section 3 reviews the oblique illumination properties. In section 4 the feature descriptors selected for characterising contrast and sharpness of the different diatom preparations analyzed are shown. Section 5 presents a quantitative evaluation study of the oblique illumination technique for different patch stop filters with different shapes and sizes. Finally, a discussion an few conclusions are drawn in the last section.

\section{Materials and methods}

Microscopic identification and counting of diatoms requires obtaining frustule suspensions free of organic matter. The frustules are the siliceous covering of the diatoms, their shape and ornamentation is the basis of the taxonomy of these algae. To empty the cell contents of the diatoms and eliminate other nonsilicified organisms, it is necessary to process the sample to an intense oxidation by hydrogen peroxide. For the observation of diatoms under optical microscopy, it is necessary to mount permanent preparations using a synthetic resin $\left(\right.$ Naphrax $\left.{ }^{\circledR}\right)$ as a medium, with a refraction index of 1.7. Sample images were captured using a low cost Brunel SP30 monocular microscope with standard Brunel DIN parfocal objectives of $60 \times(0.85$ NA) and $100 \times(1.25 \mathrm{NA})$ using LED with different wavelengths from visible to UV. For white light $(\lambda=442 \mathrm{~nm})$ a Brunel Digicam LCMOS 5 Mpixel camera was used for image acquisition (cell size $2.2 \mu \mathrm{m} \times 2.2 \mu \mathrm{m}$ ). The camera is connected to the computer through an USB2.0 connection, providing an image size of $2592 \times 1944$ pixels and the exposure time was $0.25 \mathrm{sec}$. For UV light $(\lambda=365 \mathrm{~nm})$, the Imaging Source camera DMK 72 with USB 2.0 was used. The DMK 72 is a monochrome $5 \mathrm{Mpixel}$ camera that has good sensitivity for $365 \mathrm{~nm}$. Both cameras meet the resolution limit of the corresponding objectives for the wavelengths used. Concerning the appropriateness of the microscope optics with UV light, the fewer glass components the better. Flat field objectives are not suitable for UV due to the high absorption. Glass slides and coverslips produce minor losses [28]. A centering telescope (CT) eyepiece (Bertrand lens) was used for visualising the different diffraction spectra at the back focal plane of the objective lens. Results reported in Figures 6 and 7 were obtained with a cooled Hamamatsu C9100-02 camera (CCD chip 1000x1000 pixel with $8 \mu \mathrm{m} \times 8 \mu \mathrm{m}$ cell size) attached to a Leica AF6000LX multidimensional microscope with an objective lens PL APO 40x0.75 DRY. The radius of the Airy disk produced by such objective considering the Rayleigh criterion is about $14 \mu \mathrm{m}$ what does not meet the Nyquist sampling theorem that requires two pixels per unit resolution distance (i.e. $16 \mu \mathrm{m}$ ). However such camera provides excellent sampling for 100x 1.3 NA which turns to be very suitable for fluorescence applications. The Leica microscope was used because it was the only available equipment that provides all microscopic modalities described in section 5.

\section{Oblique illumination in the context of diffraction theory}

When a transparent biological specimen is observed under a standard microscope brightfield illumination, light is retarded $\lambda / 4$ ( $\lambda=$ wavelength) with respect to the undeviated light. Therefore, there are details that are invisible in brightfield microscopy. By illuminating the specimen with oblique light a displacement in the diffraction orders is observed allowing the objective lens to capture the zero order at one side and few of the higher orders that are not captured when axial light is used. In oblique (or anaxial) illumination the substage condenser aperture is unevenly illuminated producing a differential or shadowcast of increased true resolution. For obtaining such uneven illumination, Abbe proposed to move the condenser diaphragm to one side. In fact, he recommended the use of narrow axial illuminating pencil of light instead of broad illuminating beams. This method is capable to produce directionally shadowed differential contrast images allowing to observe phase details in a similar way to differential contrast images (DIC). The main advantage of this method lies in the fact that the equipment required is cheap, because only a brightfield microscope is required. Oblique illumination can increase true resolution if higher order maxima of the specimen's diffraction pattern are captured. The way how in the practice oblique illumination is obtained is e.g. by stopping out a greater portion of the condenser's light by e.g. a crescent moon-shaped plate so the light is projected very obliquely upon the specimen.

According with Abbe $[8,11]$, the limit of resolution criterion of a microscope for axial coherent illumination is $d_{A}=\lambda / N A$ where $d$ represents the resolution power, $\lambda$ the wavelength and $N A$ the numerical aperture. From his experiments with gratings, Abbe concluded that it would be necessary to capture the first order diffracted rays at least for resolving grating details. With the development of more advanced substage condensers, oblique illumination was progressively abandoned by microscope manufacturers by the end of the nineteenth century. However, oblique illumination was reconsidered by other researchers in subsequent years and in fact the Hoffman modulation contrast technique can be interpreted as a modified extension of it [12]. On the other hand, Rayleigh considered that resolution is not only limited by diffraction but also by the contrast. The Rayleigh criterion indicates that two points can be resolved if the central maximum of the Airy disk produced by a lens lies over the minimum of the other disk. In such case, the limit of resolution is given by $d_{R}=0.61 \lambda / N A$. The Rayleigh's resolving power is less than the Abbe's criterion however the former is widely accepted. All the previous limits correspond to lateral resolution. The Rayleigh criterion along the optical axis (Zaxis) can be estimated according with $d_{R}=2 \lambda n /(N A)^{2}$, where $n$ represents the refraction index. Oblique illumination has the effect of effectively doubling the numerical aperture by allowing to transmit the zeroth- and first-order diffracted orders producing a resolution limit estimation given by $d_{\text {oblique }}=\lambda / 2 N A$ [8]. 
Very often the Abbe resolution limit is referred in many textbooks by the expression $d_{\text {oblique }}$ instead by $d_{A}$ but this is only valid when the sample was illuminated considering not only axial light but the full range of angles. For example, using ultraviolet LED light $(365 \mathrm{~nm})$ with an objective 100x $(\mathrm{NA}=1.25)$ and considering oblique illumination, the resolution limit can be reduced to $d_{\text {oblique }}=146 \mathrm{~nm}$.

Different oblique filter wheels using crescent moon, arrow, notch and circular (COL) shapes of different sizes have been generated to be attached replacing the filter holder below the substage condenser. Such 3D designs can be easily adapted to other different microscopes. Figure 1 shows a picture of four different oblique filter wheels used in this paper.

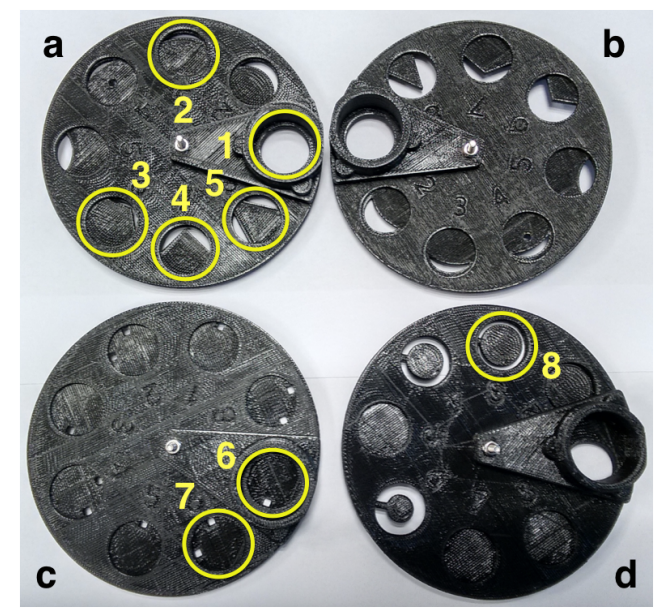

Figure 1: Oblique filter wheels generated with a 3D printer using different shapes. In wheel (a), filter 1 corresponds to brighfield illumination and filters 2-5 provide oblique illumination with the best performance both in terms of sharpness and texture contents. In wheel (c), by inserting small aperture or notch-shaped the object will be illuminated with a very oblique pencil of light. In wheel (d) circular apertures are used producing an inverted oblique "hollowcone illumination", i.e. circular oblique lighthing(COL)

There is a lack of a complete theory that provides an explanation for the appearance of the images formed with oblique illumination [8]. A simple and straightforward approach will be to consider the Abbe's theory of image formation which states that the finest details of an object depends on the pattern of diffraction located at the back focal plane of the objective lens. Figure 2 presents few illustrative examples of the different diffraction patterns observed at the back focal plane of the objective considering different oblique filters. Figure $2 b$ corresponds to brightfield axial illumination. Figure $2 \mathrm{~d}$ shows the diffraction pattern in the case of a notch filter (filter 6 from Figure 1c). Figure $2 \mathrm{f}$ corresponds to circular oblique filter COL (filter 8 from Figure 1d).

\section{Image feature characterization}

To effectively study the effect of the oblique illumination, it is required to objectively quantify the contrast and quality of the acquired images. In this paper five features were selected for image feature characterization: the Haralick's texture inertia

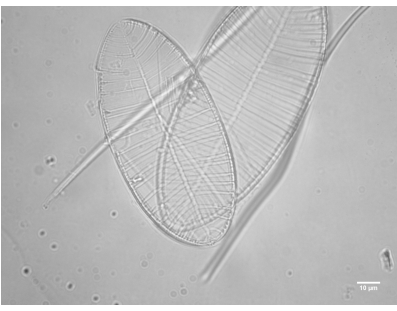

(a)

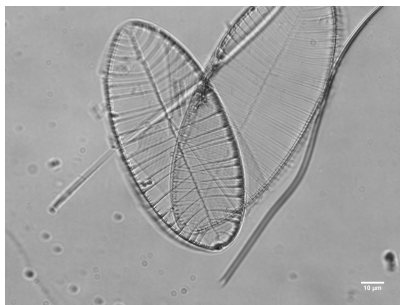

(c)

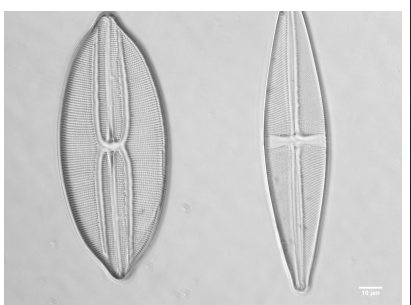

(e)

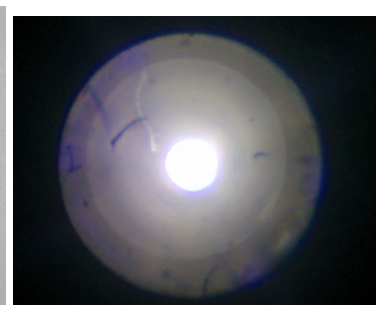

(b)

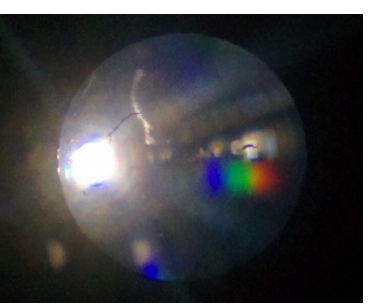

(d)

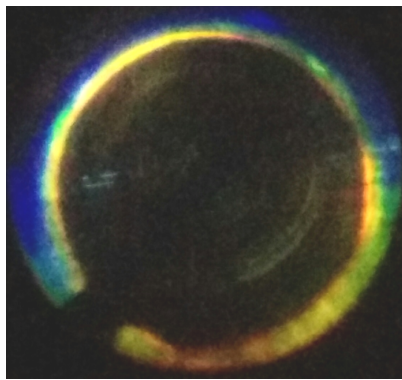

(f)
Figure 2: (a) Diatom sample image captured in brightfield microscopy; (b) Diffraction pattern created by the sample at the back focal plane of the objective. Note that mainly the zero order spectra is present (c) Diatom sample captured with oblique light using the notch filter of Figure 1c; (d) Diffraction pattern created by the sample at the back focal plane of the objective. Note how the zero order spectra has been displaced to the egde and the high diffraction orders appear at the other edge [2]; (e) Diatom image sample captured using filter 8 from Figure 1d; (f) Diffraction pattern created by the sample at the back focal plane of the objective. Note that the high diffraction orders are concentrated around the edge. Scale bars represent $10 \mu m ; \lambda=442 \mathrm{~nm}$. 
and the range filter provide good estimates for the contrast; the variance of the Laplacian as a measure of sharpness; the Angle Measure Technique spectrum (AMT) as a measure of texture complexity and the Anisotropic Quality Index (AQI) as a quality measure provided by an ideal observer [13, 15-17]. The rationale behind the selected features is to provide a complementary set of image indicators although other alternative traits are possible. The variance of the Laplacian was found particularly effective as a simple and straightforward autofocus metric [15] which can be implemented in a real-time system [29].

- Inertia $(I N E)$. Inertia or contrast is a statistics extracted from the gray level co-occurrence matrix $P(i, j)$ (GLCM) [13] that provides a measure of the second order statistical texture features. It can be interpreted as a texture descriptor to measure the intensity contrast between a pixel and its neighbours

$$
I N E=\sum_{i=0}^{G-1} \sum_{j=0}^{G-1}(i-j)^{2} P(i, j)
$$

where $\mathrm{G}$ is the number of gray levels used, and $P$ represents the GLCM of the input image $I(i, j)$. A MATLAB toolbox for computing the INE measure is available from [27]

- Local contrast (LC). Range filtering provides a measure of local contrast i.e. computes local intensity range in a neighbourhood around each pixel in an image.

$$
L C=\operatorname{mean}(\text { mean }(\operatorname{rangefilt}(I))) .
$$

where rangefilt represents the Matlab function that provides the local range of an image.

- Variance of Laplacian (VarLAP). The variance of a second derivative operator such as the Laplacian was considered as an image sharpness measure [15]. It is given by

$$
\operatorname{VarLAP}=\sum_{i=0}^{M} \sum_{j=0}^{N}[L(i, j)-\bar{L}(i, j)]^{2},
$$

where $L(i, j)$ represents the convolution of the original image $I(i, j)$ with the Laplacian operator $L A P$ :

$$
L A P=1 / 6\left[\begin{array}{ccc}
0 & -1 & 0 \\
-1 & 4 & -1 \\
0 & -1 & 0
\end{array}\right]
$$

and $\bar{L}(i, j)$ is the mean of absolute values. A MATLAB toolbox for computing the VarLAP measure is available from [25].

- Anisotropic quality index (AQI). An important texture feature is the degree of anisotropy which is directly related with the degree of coherence [18]. On the other hand, the variance of the expected directional entropy (anisotropy) called Anisotropic Quality Index (AQI) has shown to provide an excellent performance as a no reference quality measure and therefore as the response of an ideal observer.

$$
A Q I \equiv \sigma(\bar{R}(\theta))
$$

where $\bar{R}(\theta)$ represents the local pixel-wise directional entropy for the whole image [17]. A MATLAB toolbox for computing AQI for arbitrary grayscale or color images is available from [26].

- Angular Measure Technique (AMT) spectrum. AMT was proposed by R. Andrle as an alternative to fractal analysis in the context of characterising the complexity of geomorphic phenomena [19] and later extended to textured imagery [16]. AMT produces a scale-spectrum that will be useful for characterising texture complexity at all scales either with fine or coarse structures. In the case of 2D images, AMT needs to be unfolded to a $1 \mathrm{D}$ vector representation. In order to have a statistically representative measure a significant number of random points (e.g. $M=100$ points) need to be considered according with the following expression,

$$
\theta_{s}=1 / M \sum_{i=1}^{M}\left(\theta_{i}\right)_{s}
$$

where $\theta_{i}$ represents the complementary angle composed by the connecting lines between such point $i$ and the intersection of the $1 \mathrm{D}$ vector representation by a circle with radius $s$ centred in such point. The measure is based on the entire set of random points and provides an average angle or mean angle. An interesting property of the AMT spectrum is that it reflects the signal complexity on all possible scales simultaneously [16]. An ImageJ plugin for computing the AMT spectrum is available from [24].

\section{Results}

\section{Oblique light evaluation}

In this study 25 different fields of diatoms were selected. For evaluating the performance of the texture, the five features described in the previous section were computed over the above mentioned 25 fields. Figure 3 gives a quantitative account of the response of the five previously described features for brightfield illumination and in the case of the four best performing OL filters (filters 2-5 from Figure 1a). All of these measures present different dynamic range, therefore each one has been normalised by its maximum absolute value along each category. Note how all the selected OL filters provide a significant improvement for all selected indicators, including the response of an ideal observer given by the AQI measure that has shown an excellent performance as a no-reference quality measure, providing a high correlation with Mean Opinion Scores (MOS) of human observers. 


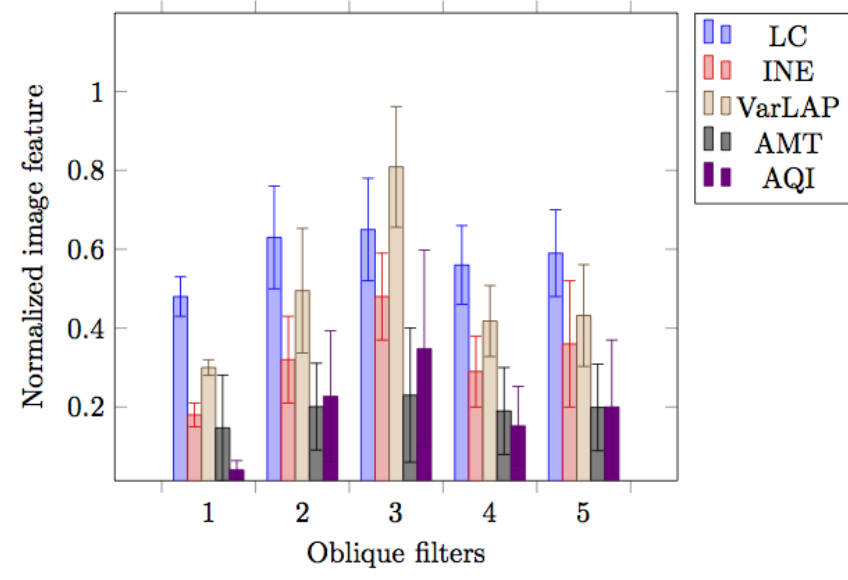

Figure 3: Local contrast, sharpness, texture complexity and quality measures corresponding to 25 image frames of diatoms by using BF filter (1) and four OL filters (2-5) as shown in Figure 1a. Note how the use of OL filters produce a steady improvement in all feature selected. Bars represent mean values and error bars standard deviation. $\lambda=442 \mathrm{~nm}$.

An additional measure of the degree of improvement obtained by the oblique light is given by the coherence image representation that can be interpreted as a combined measure of edgeness and orientation (Figure 4) [20].

Figure 5 presents an example of the use of OL and COL filters for improving contrast. Figure 5a shows the result of using a notch-shaped filter (filter 6 from Figure 1c). When the oblique illumination is oriented along the horizontal direction it allows to observe the vertical markings (striae) of the Amphipleura pellucida diatom that are separated 250nm (top specimen). The horizontal markings (striae) which are separated 200nm (bottom specimen) if the oblique illumination is oriented vertically. Finally, Figure $5 \mathrm{~b}$ presents an example of images acquired using a COL-shaped filter (filter 8 from Figure 1d) in the case of the Navicula lyra specimen. Because COL use a symmetrical hollow-cone of illumination it does not produce the strong relief found in unsymmetrical OL although in general the later improves the edges.

\section{Comparison with other microscopic modalities}

A first comparative account was performed using the five feature descriptors described in Section 4. In phase-contrast microscopy a simple way to produce OL is by slightly moving the phase-contrast condenser and adjusting the aperture diaphragm [8]. Phase contrast microscopy is a suitable technique for visualising transparent specimens. However it produces some artefacts (halos and shading off) that limits its usefulness in some applications. Halos are unresolved images with reverse contrast and shading-off is a contrast decreasing effect from the edge of a flat great area specimen (ideally shaped as a plane parallel plate) towards the center of it [8]. DIC microscopy is also a popular method for improving contrast of unstained specimens. DIC is absent of the halo effect found in phase contrast microscopy producing pseudo-relief images (but real re-

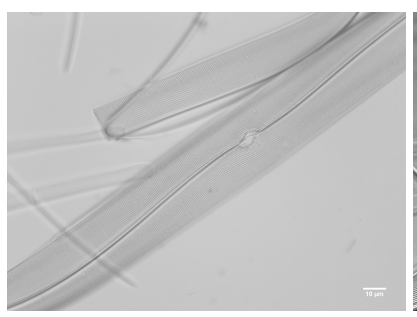

(a)

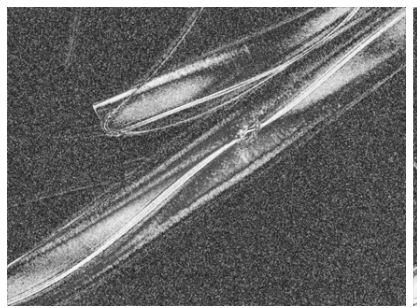

(c)

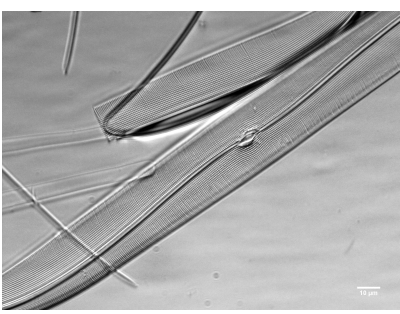

(b)

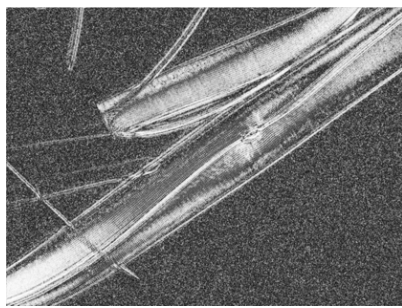

(d)
Figure 4: (a) Brigtfield image containing few diatoms; (b) Captured image using oblique filters; (c) Coherence image corresponding to Fig. 4a; (d) Same for Fig. 4b. The coherence image represents the degree of anisotropy at each point in the texture and it can be interpreted as a combined measure of edgeness and orientation. Scale bars represent $10 \mu m ; \lambda=442 \mathrm{~nm}$.

liefs in specimens of constant refractive index) that can be understood as the derivative of the optical path length defined as product of the index of refraction times the specimen thickness. Such derivative is obtained by splitting polarised light into two beams. DIC images have a somewhat high-pass appearance as if they have been acquired through highly oblique illumination. Figure 6a shows a comparative account of the different illumination modalities for the diatom Pinnularia neomajor, where the halo effect that can be observed in phase contrast (PH) and the high-pass effect can be noticed in DIC. Figure 7a presents a comparative account of the previous diatom image sample showing that both the oblique light (OL) or the combination of OL with polarisation provide a better performance for the five selected image features and simultaneously a better natural appearance in comparison with the other contrast-oriented modalities (PH and DIC). The overall mean value for the selected features are 0.79 for OL, 0.51 for DIC and 0.47 for PH. Figure $6 \mathrm{~b}$ shows a comparative account of the different illumination modalities for an histological sample (brain autopsy). Figure $7 \mathrm{~b}$ presents the same comparison for such preparation. In this case the phase-contrast modality $(\mathrm{PH})$ provides the better performance (mean value $=0.93$ ) followed by the other two modalities: OL (mean value $=0.81$ ) and DIC (mean value $=0.61)$.

\section{Resolution assessment}

A second comparison study has been accomplished by evaluating the resolution improvement provided by the use of oblique light. The spatial resolution of any imaging system can be characterized by the impulse response or point spread function (PSF). However, unlike the situation in fluorescent microscopy where the PSF is extracted using microbeads, with brightfield microscopy it is usually very difficult to directly measure the 


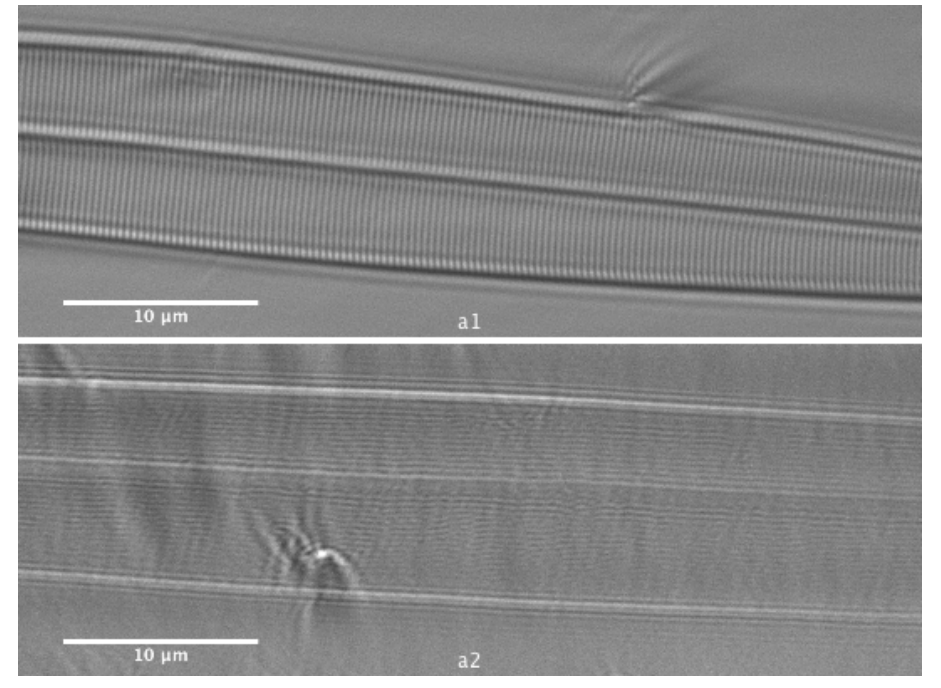

(a)

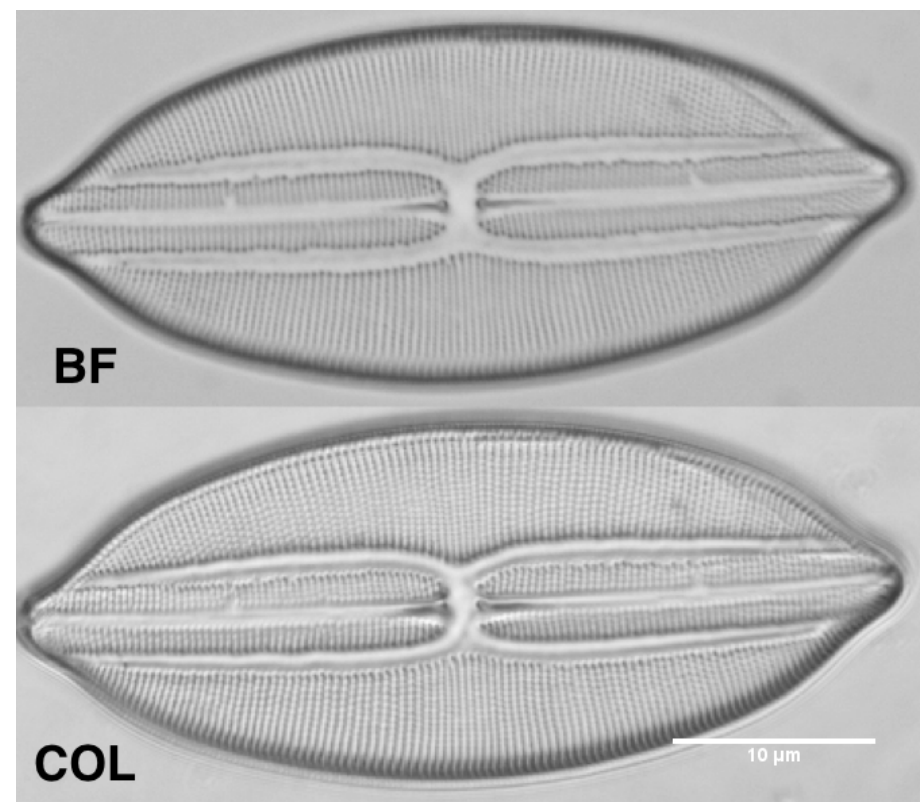

(b)

Figure 5: (a) In the diatom Amplipleura pellucida, oblique light illumination oriented along the horizontal direction allows to observe lateral markings which are separated $250 \mathrm{~nm}$ center to center (a1) and illumination oriented along the vertical direction allows to see the horizontal markings which are separated $200 \mathrm{~nm}$ (a2). Objective $100 \times$ DIN parfocal (1.25 NA); $\lambda=365 \mathrm{~nm}$. (b) Lyrella lyra diatom observed with brighfield visible light (BF) and COL. Objective $60 \times$, DIN parfocal $(0.85 \mathrm{NA}) ; \lambda=442 \mathrm{~nm}$.
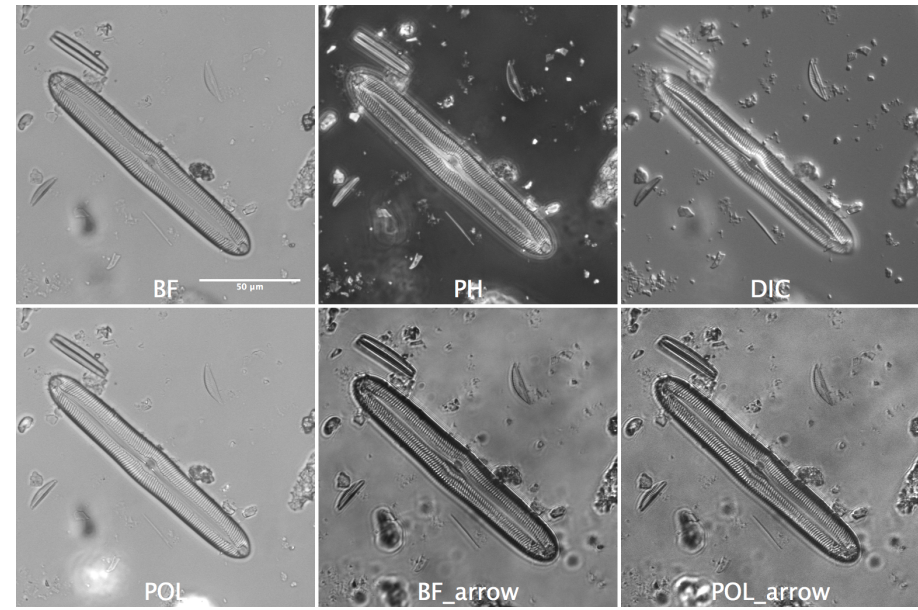

(a)
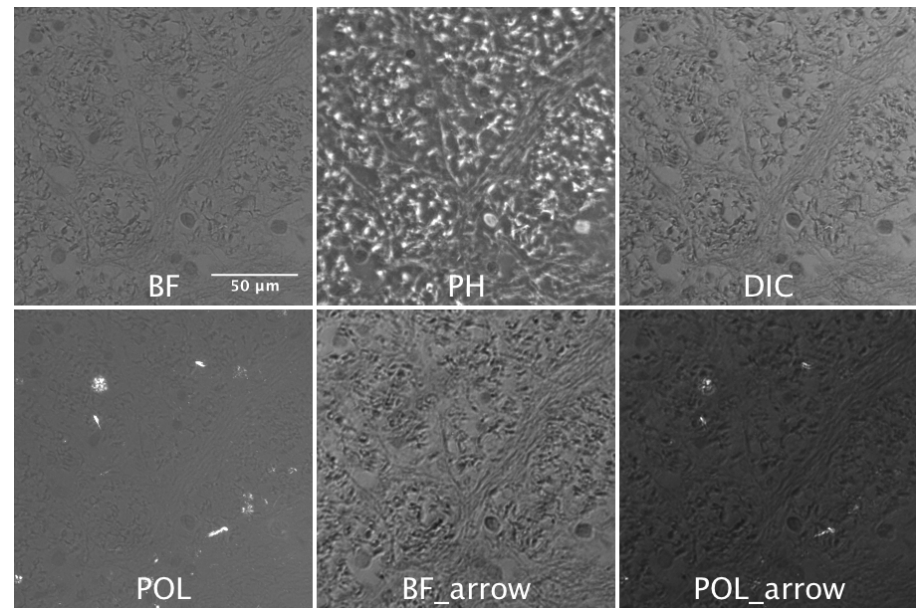

(b)

Figure 6: (a)Pinnularia neomajor diatom visualized using the following illumination modalities: $\mathrm{BF}=$ Brightfield; $\mathrm{PH}=\mathrm{Phase}$ contrast; $\mathrm{DIC}=$ Differential Interferential Contrast; $\mathrm{POL}=$ Polarization; $\mathrm{BF}_{\text {_arrow }}=\mathrm{Oblique}$ Light $(\mathrm{OL})$ and POL_arrow=OL combined with polarization; (b) Same as (a) but for a brain autopsy sample. $\lambda=450 \mathrm{~nm}$ 


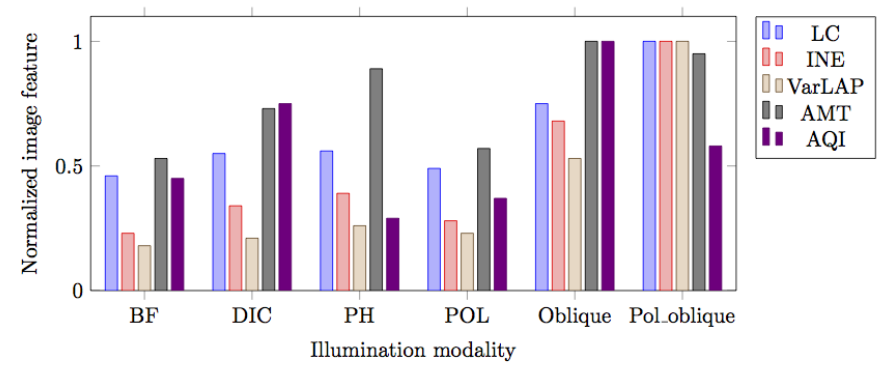

(a)

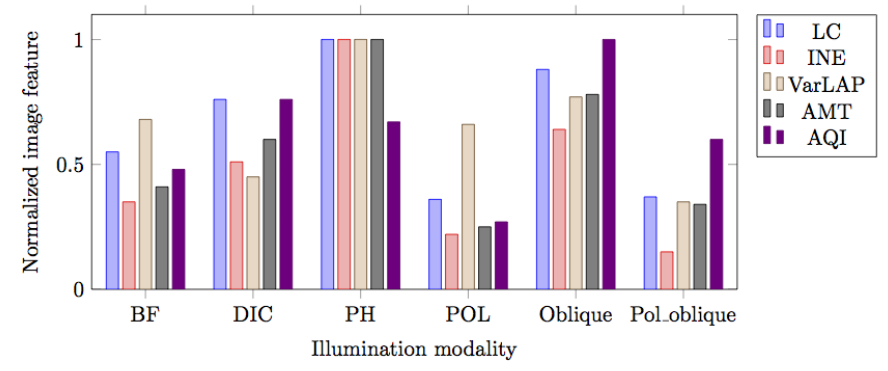

(b)

Figure 7: (a) Local contrast (LC); Inertia (INE); Sharpness(VarLAP); texture complexity (AMT) and quality estimate (AQI) values for different illumination modalities (diatom sample); (b) Same comparison corresponding to an histological sample (brain autopsy). $\lambda=450 \mathrm{~nm}$

PSF due to a weak signal contrast and low signal-to noise ratio (SNR) besides the fact that PSF width does not fit well with human perception [21]. An indirect method of PSF generation was proposed by Tadrous [22] based on a 3D deconvolution procedure through the use of a thin sample. A good alternative to the spatial resolution estimation was proposed by [21] based on the extraction of a single parameter from an edge or by the line spread function (LSF) response as the derivative of the edge response. The LSF can be considered as good approximation of the PSF in many imaging systems and it is very common to use the former as a substitute of the later. One of the main advantages is that the edge response is very simple to measure using the different commercial resolution targets available. Table 1 presents the results of the width of the LSF expressed as the the edge response to rise from $10 \%$ to $90 \%$ which provides the best single parameter measurement for the microscope resolution corresponding to the USAF resolution target. The table includes values for BF illumination and for few of the oblique filters selected in Figure 1. The resolution of a system allows to understand how the edges are blurred by the image formation. Table 1 confirms that the use of oblique illumination provides a steady resolution improvement for all the filters analyzed.
Table 1: $10 \%$ to $90 \%$ edge response using brightfield (BF) (filter \#1) and different oblique light (OL) (filters \#2-\#8) corresponding to the USAF resolution target. The units of the distance are given in lp/pixel (i.e. line pairs/pixel). The Brunel SP30 microscope with 60x objective was used.

\begin{tabular}{|c|c|c|c|c|c|c|c|}
\hline Filter & $\# 1$ & $\# 2$ & $\# 3$ & $\# 4$ & $\# 5$ & $\# 6$ & $\# 8$ \\
\hline lp/pixel & 0,005 & 0,014 & 0,047 & 0,047 & 0,0208 & 0,02 & 0,03 \\
\hline
\end{tabular}

\section{Discussion and conclusions}

Oblique illumination is an old technique that has been described by many scientists and microscopists. However, it was neglected by microscope manufacturers by the end of the nineteenth century, although in subsequent years it has been reinvented and improved. Oblique lighting is capable to produce directionally shadowed differential contrast images allowing to observe phase details in a similar way to differential contrast images (DIC) but at lower cost. In practice, oblique illumination is obtained by blocking part of the condenser's light through the use of particular patch stops with different shapes. The main advantage of OL is that the resolution of the light microscope can be increased by effectively doubling the angular aperture. Different oblique filter wheels were generated with crescent moon, arrow, notch and circular (COL) shapes using a 3D printer. A quantitative account of oblique lighting in brigthfield microscopy was presented. Several feature descriptors were selected for characterising contrast and sharpness of transparent specimens such as diatom preparations showing that OL in general provides a similar or even better performance compared to other modalities (such as PC or DIC) for distinguishing details as it has been also confirmed with the use of an ideal observer.

In relation with the application areas where the use of oblique illumination will be of interest, two different scenarios can be distinguished. First, in the case of diatoms $(n=1.43)$ where a high refractive mounting medium is used (Naphrax, $n=1.7$ ) an extraordinary great difference in refractive index can be observed between the frustules and the preparation media $(\Delta n=$ 0.27 ). When Naphrax is used as mounting medium the refractive index of such mounting exceeds the range $(n=1.334-1.518)$ of normal media used in the optical design of standard objectives. Therefore, in all common microscopic modalities the image quality will be lower than in standard preparations. OL will be a simple and cost-effective way to improve the image quality in such preparations. In other life sciences applications i.e. when living cells are examined in water or in the case of cancer or erythrocyte cells observed in a physiological medium such as blood plasma such refractive index difference $(\Delta n=0.016-0.036)$ is much smaller. The higher such refractive index difference, the better the contrast will be obtained. In the case of diatom samples both oblique light (OL) or the combination of OL with polarisation provide a better performance for the five selected image features and simultaneously a better natural appearance in comparison with the 
other contrast-oriented modalities (PH and DIC). However in the case of the histological sample analyzed (brain autopsy), the phase-contrast modality $(\mathrm{PH})$ provides the better performance (mean value $=0.93$ ) followed by the other two modalities: OL (mean value $=0.81)$ and DIC (mean value $=0.61)$. A similar performance to the later case was observed in the case of preparations with epithelial cells from the oral mucosa. For such reason, the results from the diatom samples cannot directly be transferred to other life sciences applications. However, even in such specific applications, OL is capable of visualizing native cells better than normal brightfield, what will be very useful when PH or DIC are not available in the microscope setup. Elsewhere, the use of a LED in the UV range together with a monochrome camera allow to capture images with higher resolution and therefore to discriminate diatom details that are otherwise undistinguishable with white light. Usual preparations in life sciences have not been tested with UV light and their analysis is out of scope of this paper. In summary, OL appears as a cost-effective technique both for the amateur and professional scientist that can be used as a replacement of DIC or phase contrast when resources are scarce. The Standard Tessellation Language (STL) files for 3D printing the oblique filter wheels used in this paper can be found from [10].

\section{Acknowledgements}

This work has been partially sponsored by the CTM201451907 project funded by the Spanish Ministry of Economy, Industry and Competitiveness. The authors thank Ms Gema Elvira (Center of Biological Research, CSIC, Spain) for acquiring multimodal images with the Leica AF6000LX multidimensional microscope and Dr. Lucia Gonzalez (HGUCR, Ciudad Real, Spain) for providing histological preparations.

\section{References}

[1] T. Stephanides, The microscope and the practical principles of observation, Faber and Faber, London, 1947.

[2] C. Olliver, The intelligent use of the microscope, Chapman \& Hall, London, 1947.

[3] J. Schleiden, Principles of scientific botany, Longman, London, 1849.

[4] H. Schacht, The microscope and its application to vegetable anatomy and physiology, S. Highley, London, 1853.

[5] E. Spitta, Microscopy. The construction, theory and use of the microscope, J. Murray, London, 1920.

[6] M. Francon, Progress in Microscopy, Pergamon Press, 1961.

[7] R. Allen, The microscope, Van Nostrand, Boston, 1944.

[8] R. Wayne, Light and Video Microscopy, Elsevier, Boston, second edn., 2014.

[9] W. Mathews, The use of hollow-cone illumination for increasing image contrast in microscopy, Trans. of the American Microscopical Soc. 72 (1953) 190-195.

[10] C. Sanchez, G. Cristobal, Oblique filter wheels., doi:10.6084/m9. figshare.4754260, accessed: 2017-09-19, 2017.

[11] E. Abbe, On the estimation of the aperture of the microscope, J. Roy. Microscop. Soc. Ser. II 1 (1881) 349-388.

[12] R. Hoffman, L. Gross, Modulation contrast microscope, Applied Optics 14 (1975) 1169-1176.

[13] R. Haralick, Textural features for image classification, IEEE Trans. on Systems Man and Cybernetics 6 (1973) 269-285.
[14] H. Tamura, S. Mori, T. Yamawaki, Textural features corresponding to visual perception, IEEE Trans. on Systems Man and Cybernetics 8 (1978) 460-473.

[15] J. Pech-Pacheco, G. Cristobal, J. Chamorro-Martinez, J. FernandezValdivia, Diatom autofocusing in brightfield microscopy: a comparative study, in: Proc. IEEE Conf. on Image Processing, 314-317, 2000.

[16] K. Esbensen, K. Hjelmen, K. Kvaal, The AMT approach in chemometrics-first forays, J. of Chemometrics 10 (1996) 569-590.

[17] S. Gabarda, G. Cristobal, Blind image quality assessment through anisotropy, J. Opt. Soc. Am. A 24 (2007) B42-B51.

[18] A. Rao, B. Schunck, Computing oriented texture fields, CVGIP: Graphical Models and Image Processing 53 (1991) 157-185.

[19] R. Andrle, The angle measure technique: a new method for characterizing the complexity of geomorphic lines, Math. Geology 26 (1994) 83-97.

[20] Z. Püspöki, M. Storath, D. Sage, M. Unser, Transforms and Operators for Directional Bioimage Analysis: A Survey, in: W. De Vos, S. Munck, J.-P. Timmermans (Eds.), Focus on Bio-Image Informatics, vol. 219, chap. 3, Springer, 69-93, 2016.

[21] S. Smith, The Scientist and Engineer's guide to Digital Signal Processing, Ch. 25, California Technical Publishing, San Diego, 1997.

[22] P. Tadrous, A method of PSF generation for 3D brightfield deconvolution, J. of Microscopy 237 (2010) 192-199.

[23] D. Sage, L. Donati, F. Soulez, D. Fortun, G. Schmit, A. Seitz, R. Guiet, C. Vonesch, M. Unser, DeconvolutionLab2: An Open Source Software for Deconvolution Microscopy, Methods-Image Processing for Biologists 115 (2017) 192-199.

[24] NMBU ImageJ plugins. http://bit.1y/2iMVIzf. Accessed: 201710-30.

[25] Focus measure. http://bit.1y/2zlvXgs. Accessed: 2017-10-31.

[26] AQI toolbox. http://bit.1y/2euSwDT. Accessed: 2017-10-31.

[27] Haralick texture features toolbox. http://bit.1y/2yixSCi. Accessed: 2017-10-31.

[28] P. Hobel and F.A.S. Sterrenburg. UV photomicrography of diatoms. Diatom Research, pages 1-7, 2011.

[29] S.U. Idinyang and N.A. Russell. Real-time autofocus implementation. In Functional Optical Imaging, 2011. doi : 10.1109/FOI . 2011.6154844. 